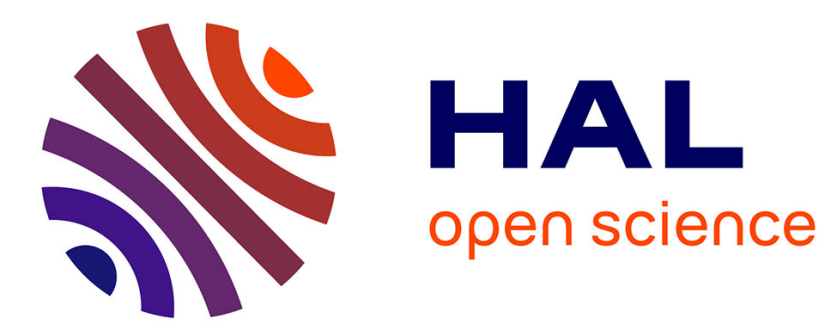

\title{
Atézolizumab et bévacizumab - carcinome hépato-cellulaire non résécable en première ligne
}

Marie-Sophie Minot-This, Julien Edeline

\section{To cite this version:}

Marie-Sophie Minot-This, Julien Edeline. Atézolizumab et bévacizumab - carcinome hépatocellulaire non résécable en première ligne. Bulletin du Cancer, 2021, 108 (2), pp.139-140. 10.1016/j.bulcan.2020.11.011 . hal-03184177

\section{HAL Id: hal-03184177 https: / hal.sorbonne-universite.fr/hal-03184177}

Submitted on 29 Mar 2021

HAL is a multi-disciplinary open access archive for the deposit and dissemination of scientific research documents, whether they are published or not. The documents may come from teaching and research institutions in France or abroad, or from public or private research centers.
L'archive ouverte pluridisciplinaire HAL, est destinée au dépôt et à la diffusion de documents scientifiques de niveau recherche, publiés ou non, émanant des établissements d'enseignement et de recherche français ou étrangers, des laboratoires publics ou privés. 
Titre :

Nouvelle AMM : atézolizumab et bévacizumab - Carcinome hépato-cellulaire non résécable en première ligne.

New approval : atezolizumab and bevacizumab for first line of unresecable hepatocellular carcinoma.

Auteurs : Marie-Sophie Minot-This, Julien Edeline

\section{Mots-clefs :}

Carcinome hépato-cellulaire - Hepatocellular carcinoma

Anti-PD-L1 (Programmed death-ligand 1)

Inhibiteurs des points de contrôle immuns - Immune checkpoint inhibitors

Anti-VEGF (Vascular Endothelial Growth Factor)

Anti-angiogènes - antiangiogenics

L'incidence du carcinome hépato-cellulaire $(\mathrm{CHC})$ en France augmente, avec actuellement environ 7000 nouveaux cas par an. Le traitement de référence en première ligne systémique est un inhibiteur de tyrosine kinase multicible antiangiogène, soit le sorafénib ${ }^{1}$ soit le lenvatinib (non remboursé en France) ${ }^{2}$, qui ont permis une amélioration modeste de la survie globale, au prix d'effets indésirables non négligeables. Le CHC est le premier cancer digestif pour lequel l'immunothérapie va trouver une place.

L'atézolizumab (Tecentriq $^{\odot}$ ) est un anticorps monoclonal humanisé dirigé contre le PD-L1. Associé au bévacizumab (anticorps anti-VEGF), son efficacité a été démontrée dans I'essai multicentrique international de phase III IMbrave $150^{3} .501$ patients, suivis pour un $\mathrm{CHC}$ non résécable, non accessible à un traitement loco-régional ou métastatique, en première ligne de traitement systémique, ont été randomisés (2:1) en ouvert pour recevoir l'association atézolizumab-bévacizumab ou le sorafénib. L'association atezolizumabbevacizumab améliorait significativement les deux co-critères de jugement principaux : survie globale (SG) à 12 mois de 67,2\% (Intervalle de Confiance (IC) à 95\% 61\%-73\%) contre 54,6\% (IC95 45\%-64\%), avec un Hazard Ratio (HR) de 0,58 (IC95 0,42-0,79; $p<0,001$ ) ; et survie sans progression (SSP) médiane de 6,8 mois (IC95 5,7-8,3) contre 4,3 mois $(4-5,6)$, avec un HR de 0,59 (IC95 0,47-0,76; $p<0,001$ ), après un suivi médian cependant encore court de 8,6 mois. En critère de jugement secondaire, le taux de réponse objective selon RECIST était significativement augmenté à $27,3 \%$ (IC95 22,5-32,5) contre 11,9\% (IC95 7,418).

Des effets secondaires sévères (grades 3-4) sont survenus plus fréquemment dans le groupe traité par la bithérapie atézolizumab-bévacizumab que dans le groupe sorafénib (38\% contre $30,8 \%)$, entraînant ainsi plus d'arrêts de traitement (15,5\% contre $10,3 \%)$. Les plus fréquents sous bithérapie étaient l'hypertension artérielle (HTA) et la protéinurie, et sous sorafénib l'HTA, la diarrhée et le syndrome main-pied. Les toxicités induites par la bithérapie impactent donc moins la vie quotidienne des patients, comme cela a été 
démontré dans les résultats secondaires de l'étude après analyse des auto-questionnaires de qualité de vie : la bithérapie permettait de retarder de manière très importante le délai d'apparition d'une détérioration de la qualité de vie, qui survenait avec une médiane de 11,2 mois pour la bithérapie contre 3,6 mois avec le sorafénib (HR 0,63 IC 0,46-0,85).

Les résultats très clairs, cliniquement significatifs, sont donc en faveur à la fois d'une meilleure efficacité importante et d'une meilleure tolérance. Il est certain que l'association deviendra le standard de première ligne. La demande d'AMM de l'association atézolizumabbévacizumab concerne donc les $\mathrm{CHC}$ non résécables ou métastatique en première ligne de traitement systémique. La posologie est de $1200 \mathrm{mg}$ d'atézolizumab et de $15 \mathrm{mg} / \mathrm{kg}$ de bévacizumab en perfusions intraveineuses toutes les 3 semaines. II faudra que les oncologues digestifs apprennent à gérer la toxicité des immunothérapies, s'appuyant sur l'expérience de leurs collègues. Il faudra aussi ne pas oublier le bilan standard de la cirrhose, notamment la fibroscopie, pour éviter les hémorragies digestives. L'atézolizumab est la première immunothérapie à avoir prouvé un bénéfice clinique dans le $\mathrm{CHC}$. Deux essais ayant testé des anti-PD-1, le nivolumab et le pembrolizumab, en monothérapie dans le $\mathrm{CHC}$ avaient échoué à montrer un bénéfice en survie, respectivement en première ligne vs sorafenib et deuxième ligne vs placebo ${ }^{4,5}$. Dans la phase $\mathrm{lb} \mathrm{GO} 30140^{6}$, il avait été démontré que l'atézolizumab présentait de meilleurs résultats d'amélioration de survie sans progression lorsqu'il était associé au bévacizumab plutôt qu'en monothérapie. II semble donc que ce soit l'association immunothérapie-antiangiogène qui soit plus pertinente que les anti-PD-(L)1 en monothérapie dans le CHC. Par ailleurs, d'autres associations intéressantes sont en attente des résultats de leur phase 3 : les associations immunothérapie-antiangiogènes lenvatinib-pembrolizumab et cabozantinib-nivolumab, et les associations anti-PD-L1-anti-CTLA-4 durvalumab-tremelimumab. Enfin, de nombreux essais d'immunothérapie sont ouverts et se développent pour démontrer l'intérêt à un stade plus précoce de la maladie, en association ou en adjuvant de la chimio-embolisation, la radiofréquence ou la résection.

\section{Références}

1. Llovet JM, Ricci S, Mazzaferro V, Hilgard P, Gane E, Blanc J-F, et al. Sorafenib in advanced hepatocellular carcinoma. N Engl J Med. 24 juill 2008;359(4):378- 90.

2. Kudo M, Finn RS, Qin S, Han K-H, Ikeda K, Piscaglia F, et al. Lenvatinib versus sorafenib in first-line treatment of patients with unresectable hepatocellular carcinoma: a randomised phase 3 non-inferiority trial. Lancet Lond Engl. 24 2018;391(10126):1163- 73 .

3. Finn RS, Qin S, Ikeda M, Galle PR, Ducreux M, Kim T-Y, et al. Atezolizumab plus Bevacizumab in Unresectable Hepatocellular Carcinoma. N Engl J Med. 14 mai 2020;382(20):1894- 905.

4. Yau T, Park JW, Finn RS, et al. Check-Mate 459: a randomized, multi-center phase 3 study of nivolumab (NIVO) vs sorafenib (SOR) as first-line (1L) treatment in patients with advanced hepatocellular carcinoma (aHCC). Ann Oncol 2019;30:Suppl 5:v874-v875. 
5. Finn RS, Ryoo BY, Merle P, Kudo M, et al. Results of KEYNOTE-240: phase 3 study of pembrolizumab (Pembro) vs best supportive care (BSC) for second line therapy in advanced hepatocellular carcinoma (HCC). Journal of Clinical Oncology 2019 37:15_suppl, 4004-4004.

6. Lee MS, Ryoo B-Y, Hsu C-H, et al. Randomised efficacy and safety results for atezolizumab (ATEZO) + bevacizumab (BEV) in patients (pts) with previously un- treated, unresectable hepatocellular car- cinoma (HCC). Ann Oncol 2019;30:Suppl 5:v875. 